Published in final edited form as:

Nat Immunol. 2015 February ; 16(2): 170-177. doi:10.1038/ni.3058.

\title{
A new class of highly potent, broadly neutralizing antibodies isolated from viremic patients infected with dengue virus
}

\author{
Wanwisa Dejnirattisai ${ }^{\# 1}$, Wiyada Wongwiwat ${ }^{\# 1}$, Sunpetchuda Supasa ${ }^{\# 1,2,3}$, Xiaokang \\ Zhang ${ }^{4,5}$, Xinghong Dai ${ }^{6}$, Alexander Rouvinski ${ }^{4,5}$, Amonrat Jumnainsong ${ }^{1,7}$, Carolyn \\ Edwards ${ }^{1}$, Nguyen Than Ha Quyen ${ }^{8}$, Thaneeya Duangchinda ${ }^{9}$, Jonathan M Grimes ${ }^{10,11}$, \\ Wen-Yang Tsai ${ }^{12}$, Chih-Yun Lai ${ }^{12}$, Wei-Kung Wang ${ }^{12}$, Prida Malasit $^{2,9}$, Jeremy Farrar ${ }^{8}$, \\ Cameron P Simmons $^{8,13}$, Z Hong Zhou ${ }^{6}$, Felix A Rey ${ }^{4,5}$, Juthathip Mongkolsapaya ${ }^{1,2}$, and \\ Gavin R Screaton ${ }^{1}$
}

${ }^{1}$ Division of Immunology and Inflammation, Department of Medicine, Faculty of Medicine, Hammersmith Campus, Imperial College, London, UK ${ }^{2}$ Dengue Hemorrhagic Fever Research Unit, Office for Research and Development, Faculty of Medicine, Siriraj Hospital, Mahidol University, Bangkok, Thailand ${ }^{3}$ Graduate Program in Immunology, Department of Immunology, Faculty of Medicine, Siriraj Hospital, Mahidol University, Bangkok, Thailand ${ }^{4}$ Institut Pasteur, Département de Virologie, Unité de Virologie Structurale, Paris, France ${ }^{5}$ CNRS UMR 3569 Virologie, Paris, France ${ }^{6}$ Department of Microbiology, Immunology and Molecular Genetics and California NanoSystems Institute, University of California Los Angeles, Los Angeles, California, USA ${ }^{7}$ The Centre for Research and Development of Medical Diagnostic Laboratories, Faculty of Associated Medical Sciences, Khon Kaen University, Khon Kaen, Thailand ${ }^{8}$ Oxford University Clinical Research Unit, Wellcome Trust Major Overseas Program, Hospital for Tropical Diseases, Ho Chi Minh City, Viet Nam ${ }^{9}$ Medical Biotechnology Unit, National Center for Genetic Engineering and Biotechnology, National Science and Technology Development Agency, Pathumthani, Thailand ${ }^{10}$ Division of Structural Biology and Oxford Protein Production Facility, Wellcome Trust Centre for Human Genetics, University of Oxford, Oxford, UK ${ }^{11}$ Science Division, Diamond Light Source, Diamond House, Harwell Science and Innovation Campus, Didcot, Oxfordshire, UK ${ }^{12}$ Department of Tropical Medicine, Medical Microbiology and Pharmacology, John A. Burns School of Medicine, University of Hawaii at Manoa, Honolulu, Hawaii, USA ${ }^{13}$ Department of Microbiology and Immunology, University of Melbourne, Carlton, Victoria, Australia

\footnotetext{
(C) 2015 Nature America, Inc. All rights reserved.

Reprints and permissions information is available online at http://www.nature.com/reprints/index.html.

Correspondence should be addressed to G.R.S. (g.screaton@imperial.ac.uk) or J.M. (j.mongkolsapaya@imperial.ac.uk)..

Accession codes. EMDataBank: cryo-EM DENV-2-Fab 747(4)B7, EMD-2818.

Note: Any Supplementary Information and Source Data files are available in the online version of the paper.

COMPETING FINANCIAL INTERESTS

The authors declare competing financial interests: details are available in the online version of the paper.

AUTHOR CONTRIBUTIONS

J.M. and G.R.S. conceived the experiments; Z.H.Z. and F.A.R. conceived the cryo-EM experiments; N.T.H.Q., J.F. and C.P.S. provided specimens; W.D., W.W., S.S., A.J., C.E. and T.D. generated human mAbs; W.D., S.S. and T.D. prepared viral stocks; W.D., S.S., A.J. and C.E. identified cross-reactivity and protein recognition of mAbs; W.D., S.S. and A.J. carried out neutralization, ADE and binding assay; P.M. provided reagents; W.D., S.S., W.W., J.M.G., W.-Y.T., C.-Y.L. and W.W. mapped epitopes; W.-K.W. mapped epitopes; A.R. produced Fab 747(4)B7; X.Z. and X.D. determined the cryo-EM structure; J.M., W.W. and C.E. analyzed antibody clonality; and W.D., J.M. and G.R.S. wrote the paper.
} 
\# These authors contributed equally to this work.

\section{Abstract}

Dengue is a rapidly emerging, mosquito-borne viral infection, with an estimated 400 million infections occurring annually. To gain insight into dengue immunity, we characterized 145 human monoclonal antibodies (mAbs) and identified a previously unknown epitope, the envelope dimer epitope (EDE), that bridges two envelope protein subunits that make up the 90 repeating dimers on the mature virion. The mAbs to EDE were broadly reactive across the dengue serocomplex and fully neutralized virus produced in either insect cells or primary human cells, with $50 \%$ neutralization in the low picomolar range. Our results provide a path to a subunit vaccine against dengue virus and have implications for the design and monitoring of future vaccine trials in which the induction of antibody to the EDE should be prioritized.

Dengue is a mosquito-borne systemic viral infection caused by any of four antigenically related dengue viruses (serotypes DENV-1, DENV-2, DENV-3 and DENV-4) ${ }^{1}$, which differ by $30-35 \%$ in amino acid sequence. Infection with one serotype leads to lifelong protection against that serotype but not against the other serotypes. There are estimated to be 400 million dengue infections annually, of which approximately one quarter are symptomatic ${ }^{2}$. Meanwhile, the geographical spread of DENV continues to widen, threatening the southern United States and Australia, and there is also concern about possible spread to southern Europe $^{2}$. The more severe cases can develop dengue hemorrhagic fever, which can lead to shock, hemorrhage and death. The primary pathogenic lesion in dengue hemorrhagic fever is a profound vascular leak that occurs at the time of viral clearance, which has led some to suggest it is an immunopathology driven by $\mathrm{T}$ cells ${ }^{3,4}$.

There is epidemiological evidence that severe disease is more likely to occur during a secondary infection (with an unrelated serotype) than during the first or primary infection with DENV ${ }^{5}$. Antibody-dependent enhancement (ADE) ${ }^{6}$ has been proposed to explain the increase in severity seen on secondary infection. This hypothesis states that antibody generated against the primary infecting DENV serotype will not be of sufficient avidity or concentration to neutralize the secondary serotype but will still opsonize the virus ${ }^{6}$. Opsonized virus can then be targeted for Fc receptor-mediated uptake into monocytes or macrophages, which leads to enhanced infection and drives greater virus production.

The enhancement of disease upon secondary infection and the need to protect against four diverse serotypes sets a high bar for vaccines, which are urgently needed to protect against this rapidly emerging disease ${ }^{2}$. Most vaccines against DENV in development aim to raise virus-neutralizing antibodies, and the DENV envelope (E) protein, which coats the virus, is the main focus of this effort ${ }^{7}$. A trial of a tetravalent live attenuated vaccine against DENV resulted in an overall vaccine efficacy of $30.2 \%$ (confidence interval, 13.4-56.6), with almost no efficacy against DENV-2, despite its stimulating the production of antibodies with in vitro neutralizing activity against all four serotypes ${ }^{8}$. A second trial also showed poor efficacy against DENV-2 infection ${ }^{9}$. There is a pressing need to investigate the human antibody response to naturally acquired DENV infection, as well as after vaccination, to understand the correlates of protective immunity. 
The recognition of DENV particles by antibodies is complicated by several different compositions and conformations of the virus glycoprotein shell that are displayed at different phases of the virus life cycle ${ }^{10,11}$. The immature viral particle has a full complement of precursor membrane protein (prM) in a 1:1 association with E protein. In an environment of neutral $\mathrm{pH}$, such as in the endoplasmic reticulum lumen in which the particles bud, the immature virion displays a characteristic appearance of 60 spikes, each a heterohexamer (usually referred to as a 'trimer') made up of three prM and three $\mathrm{E}$ proteins ${ }^{10-13}$. Exposure to low $\mathrm{pH}$ in the Golgi results in a transition at the virus surface in which trimers dissociate and the individual subunits reassociate as dimers, displaying a smooth herringbone lattice of 90 dimers of E protein ('E dimers'). In these particles, prM is bound at the E-dimer interface, covering the fusion loop and exposing a cleavage site for the host protease furin, which resides in the trans-Golgi network. Following furin-mediated cleavage of prM, mature viral particles are released into the environment; these lose the precursor moiety of prM that results from cleavage by furin. The release of prM primes the particle to become infectious, reacting to the acidic $\mathrm{pH}$ of endosomes to induce fusion of viral and endosomal membranes ${ }^{14,15}$. An important and additional level of complexity is that the cleavage of prM is frequently incomplete, which leads to a population of partially mature viruses with varying degrees of cleavage ${ }^{16}$. Viruses containing large amounts of prM are not infectious, whereas viruses with lower levels of prM are still infectious; furthermore, it has been postulated that the noninfectious particles with large amounts of prM can be driven to infect by $\mathrm{ADE}^{17}$. Expansion of smooth mature particles into a bumpy form upon exposure to temperatures above $34^{\circ} \mathrm{C}$, in which the $\mathrm{E}$ dimers rearrange with respect to each other, has also been described for DENV-2 virions ${ }^{18,19}$.

Here we examined a large panel of human mAbs that target DENV E protein, which allowed us to delineate the antigenic landscape during natural dengue infection. We have identified a new class of highly potent and broadly reactive antibodies able to neutralize multiple DENV serotypes. Antibodies directed against the envelope dimer epitope (EDE) were the only class of antibody in this study able to neutralize DENV produced in primary human cells.

\section{RESULTS}

\section{Generation of mAb to dengue}

We used blood samples from seven hospitalized patients (Supplementary Table 1) to produce human mAbs that reacted to DENV E protein ${ }^{20,21}$. We sorted plasmablasts $\left(\mathrm{CD} 3{ }^{-} \mathrm{CD} 20^{\mathrm{lo}} \mathrm{CD} 19^{+} \mathrm{CD} 27^{\mathrm{hi}} \mathrm{CD} 38^{\text {hi }}\right.$ or $\mathrm{CD} 3{ }^{-} \mathrm{CD} 20^{-} \mathrm{CD} 19^{+} \mathrm{CD} 27^{\mathrm{hi}} \mathrm{CD} 38^{\text {hi }}$ ) from peripheral blood and, by enzyme-linked immunospot assay, demonstrated that $50-90 \%$ of these cells secreted antibody to DENV (anti-DENV), consistent with reported frequencies ${ }^{22,23}$. We amplified sequences encoding heavy and light chains from single-cell cDNA and cloned these into expression vectors, and produced recombinant mAb via transfection into 293T human embryonic kidney cells. We produced 145 human mAbs; as shown by enzyme-linked immunosorbent assay (ELISA), 84\% reacted to all four DENV serotypes, $13 \%$ reacted to two or three serotypes and only $3 \%$ reacted to a single serotype (Fig. 1a).

For the initial antibody screen, we used ELISA of captured whole virions from each of the four serotypes, rather than recombinant protein or fixed cells, to ensure we obtained a fully 
representative panel of antibodies. Only $57 \%$ of the mAbs reacted to DENV E protein, as shown by immunoblot analysis (Fig. 1a). The mAbs that were not reactive by immunoblot analysis $\left(\mathrm{IB}^{-}\right.$) also failed to react to recombinant $\mathrm{E}$ protein when assessed by ELISA (data not shown). This property allowed us to categorize the mAbs into two broad groups: those that were reactive by immunoblot analysis $\left(\mathrm{IB}^{+}\right)$, and those that recognized only an epitope present on the intact virion that was $\mathrm{IB}^{-}$. Most of the $\mathrm{IB}^{+} \mathrm{mAbs}$ were fully cross-reactive among the four virus serotypes, whereas among the $\mathrm{IB}^{-} \mathrm{mAbs}, 41$ of 62 were fully crossreactive, with a further 17 of 62 reacting to DENV-1, DENV-2 and DENV-3 (Fig. 1b). These findings showed that the presence of a large group of antibodies that reacted to complex conformational epitopes on the DENV virion were produced as part of the human antibody response to natural infection with DENV.

\section{Epitope mapping of anti-DENV}

The mature DENV virion contains 180 E proteins that form 90 dimers, which are arranged through icosahedral symmetry to form a smooth glycoprotein shell ${ }^{24}$ (Supplementary Fig. 1). To gain more insight into the epitopes recognized by the mAbs, we created $65 \mathrm{DENV}$ virus-like particles (VLPs) that contained alanine substitutions at solvent-exposed residues predicted from available structural studies to be on the virion surface ${ }^{10,24,25}$. We captured VLPs onto ELISA plates coated with anti-prM and assessed reactivity to the panel of mAbs by ELISA. All VLPs contained similar amounts of prM, which was consistent with the relatively large prM content of virus produced in 293T cells and Vero African green monkey kidney cells (described in more detail below).

We screened the mutant VLPs against the 145 mAbs by ELISA ${ }^{17}$. We deemed relevant those substitutions that resulted in a reduction in antibody binding of $>80 \%$. For clarification, VLPs are not homogeneous in structure, and most are smaller in size than infectious DENV virions. The fraction of smallest radius has been characterized structurally and has been shown to be composed of $30 \mathrm{E}$ dimers, which display an arrangement different from that of the $90 \mathrm{E}$ dimers observed on infectious, mature tick-borne encephalitis virions $^{26}$. The different packing of E dimers in the VLP might therefore disrupt some complex quaternary epitopes formed between adjacent dimers in the lattice of E proteins; fortunately, however, all mAbs in this study bound to the control, non-mutant VLP, which confirmed the utility of VLPs for mapping the epitopes of this antibody panel.

With the VLP panel, we assigned 112 of $145 \mathrm{mAbs}$ an epitope on E protein to which they bound (Fig. 2a and Supplementary Table 2). Thirty-three mAbs, all of which reacted to E protein by immunoblot analysis, remained unmapped with the mutant VLP panel, which may have reflected the fact that a diffuse epitope relies on the sum of many interactions or that the change to alanine was too conservative to disrupt antibody binding. These mapping results delineated a previously unknown conformationally sensitive epitope for anti-DENV (described below).

\section{mAbs to the fusion loop epitope}

Studies of mouse mAbs raised against DENV have described a major class of antibodies that target the fusion loop epitope (FLE) centered on the hydrophobic fusion peptide on $\mathrm{E}$ 
protein used by the virus to fuse viral and host cell membranes in the acidic endosomal environment. Trp101 has been shown to be a key residue for the binding of various mAbs to FLE ${ }^{27-31}$. Of the $83 \mathrm{mAbs}$ that bound to the E protein, as assessed by immunoblot analysis, 46 were sensitive to the substitution of Trp101 (Fig. 2a). Of those 46 FLE-specific mAbs, 40 were sensitive to the substitution of Trp101 in isolation, whereas other mAbs were also sensitive to the substitution of Gly106 and Leu107 (data not shown).

A crystal structure of mAb E53 to FLE bound to E protein from West Nile virus has shown contacts with residues 104-110, but not Trp101, and with residues 74-79 in the BC loop, which lies near the fusion loop ${ }^{32}$. Only two of the FLE-specific mAbs described here were sensitive to changes in the $\mathrm{BC}$ loop; binding was lost when amino acids 76-79 were changed to alanine (data not shown), similar to $1 \mathrm{C} 19$, a published cross-reactive antibody to dengue that binds to the $\mathrm{BC}$ loop ${ }^{30}$.

\section{Conformationally sensitive mAbs to DENV}

We categorized the $\mathrm{IB}^{-} \mathrm{mAbs}$ into five distinct subgroups on the basis of their pattern of reactivity to the VLP mutants (Fig. 2a and Supplementary Table 2). Most $\mathrm{IB}^{-} \mathrm{mAbs}$ were sensitive to changes in fusion loop residue Trp101 (W101A) but are not to be confused with the classic, linear fusion loop-specific antibodies described above, as the epitope is much more extensive with additional determinants on domains I, II and III. Most of the $\mathrm{IB}^{-} \mathrm{mAbs}$ reacted to EDE, and we subcategorized these into two distinct subgroups: those that recognized E dimer-dependent epitope 1 (EDE1 mAbs), and those that recognized EDE2 (EDE2 mAbs) (Fig. 2a and Supplementary Table 2). These two subgroups could be distinguished by sensitivity to changes at residues 153 and 155 in EDE2 that disrupt an Nlinked glycosylation site. Twelve mAbs constituted three further subgroups with different epitopes and function: IB-3 mAbs were similar to EDE1 mAbs but were also sensitive to changes at Leu107 and Lys295; IB-4 mAbs were not sensitive to changes at Trp101; and IB-5 mAbs constituted a group of five mAbs that bound to the fusion loop Trp101 only, but the epitope was recapitulated only on intact virions. The data from the alanine scan delineated two related groups of antibodies, EDE1 mAbs and EDE2 mAbs, with a previously undescribed mode of binding to the DENV virion.

\section{mAbs to EDE bind across the E dimer interface}

Mapping the alanine-scanning results onto the structure of a previously determined DENV-2 E dimer ${ }^{25}$ indicated that EDE is a complex epitope that could be contained within an E dimer or could be shared by adjacent $\mathrm{E}$ dimers at the surface of the virion (Fig. 2b). We therefore analyzed DENV-2 particles in complex with the antigen-binding fragment (Fab) of one of the EDE2 mAbs (747(4)B7) by cryo electron microscopy (cryo-EM) and obtained a three-dimensional reconstruction at sub-nanometer resolution (Fig. 2c). The reconstruction showed density for bound Fab of mAb 747(4)B7 (Fab 747(4)B7), which indicated that it bound at an intradimer epitope and not across dimers. This was better demonstrated with the density corresponding to bound Fab over the underlying organization of the E dimers on the glycoprotein shell after superposition with the $3.5 \AA$ cryo-EM structure of DENV-2 (ref. 24), with three $\mathrm{E}$ polypeptides of the icosahedral asymmetric unit presented here in different 
colors and as solid surfaces (Fig. 2d). The same superposition with the density map of the complex can also be presented as a transparent mesh (Supplementary Fig. 2).

The distribution of $\mathrm{E}$ and $\mathrm{M}$ subunits in a herringbone pattern was such that there were two different types of dimers: red-red dimers related by the icosahedral twofold axis, and cyanyellow dimers related by a local twofold axis (Fig. 2d). The Fab density on the E dimer was at the $\mathrm{E}$ dimer interface, over the fusion loop, and matched the clusters of mutations from the alanine scanning experiments (Fig. 2e). We extracted dimer from the cryo-EM structure of the virion-Fab 747(4)B7 complex and have presented it along the molecular local twofold axis (Fig. 2b).

In the virus particle, we found that the density of the Fab bound to the dimer related by the icosahedral twofold axes of the particle (red-red dimer) was somewhat weaker than that of the Fab bound to the dimer sitting at a local twofold axis (cyan-yellow dimer), the density of which was comparable to that of the E-protein shell on the particle (Fig. 2d). Because the epitopes have the same chemical composition, this indicated that they were not exposed identically on the two types of dimers in the particle. The X-ray structure of Fab 747(4)B7 in complex with recombinant protein E from DENV-2 to a resolution of $3 \AA$ has been published, with details of the interactions at the E dimer interface ${ }^{33}$. That publication also describes similar structures of the $\mathrm{E}$ protein in complex with three other antibodies from both the EDE1 cluster and EDE2 cluster $^{33}$, which confirms that the antibodies of the two subgroups bind in a similar way. The X-ray structures reveal an entirely novel mode of binding, with antibodies bridging the E dimer interface distinct from other conformationsensitive antibodies ${ }^{34-37}$.

The EDE overlapped with the FLE, which led us to compare the frequency of mAbs reacting to the FLE and those reacting to the EDE in samples from individual patients. We found skewed repertoires showing a 'preference' for either FLE or EDE (Fig. 3). This immunodominance of recognition in a patient was unexpected, as the antibody responses to FLE or EDE were polyclonal, which would indicate that the 'choice' between the two responses in a patient was not simply being driven stochastically by shielding of the epitope by whichever of the antibodies to FLE or EDE were the first to bind.

\section{Neutralization potency of mAbs to EDE and FLE}

We performed neutralization assays of DENV produced in C6/36 insect cells for three mAbs from each of the FLE and EDE groups (Fig. 4). Both mAbs to FLE and mAbs to EDE were broadly neutralizing against all four virus serotypes. The EDE2 mAbs (defined as those sensitive to glycosylation at Asn153: mAb 747(4)A11 and mAb 747(4)B7) showed lower activity against DENV-4, which is related to the fact that the strain we used lacks the glycan at position 153. This is a natural variant, but for most DENV-4 strains, glycosylation at this position is maintained. These data showed that the EDE defined the binding site for a group of antibodies that were broadly reactive across the DENV serocomplex.

\section{Neutralization of DENV produced in insect or mammalian cells}

The mAbs to EDE reported here were among the most potent and broadly reactive mAbs to DENV, with a $50 \%$ neutralization titer $\left(\mathrm{NT}_{50}\right)$ as low as $3.3 \times 10^{-11} \mathrm{M}$. During a natural 
infection with DENV, the host is presented with two forms of the virus: the initial exposure is to DENV produced in cells of the insect vector, whereas DENV produced in human cells drives subsequent rounds of infection and represents the vast bulk of DENV encountered during infection. Viruses produced in these two different types of host cell differ antigenically. To examine the neutralization of these two different viral forms, we compared neutralization of DENV-2 produced in C6/36 insect cells (C6/36-DENV) with that of virus produced in human monocytederived dendritic cells (DCs) (DC-DENV), which are thought to be infected after injection of DENV into the skin from the mosquito bite and to be a site of DENV replication in the infected human host ${ }^{38}$.

Of the $83 \mathrm{IB}^{+} \mathrm{mAbs}$, we mapped 46 to the FLE, and 37 recognized an as-yet-unmapped binding site (Supplementary Table 2). None of the $83 \mathrm{IB}^{+} \mathrm{mAbs}$ fully neutralized DCDENV, even at high concentration, with only one neutralizing DC-DENV to $>80 \%$ at a concentration of $5 \mu \mathrm{g} / \mathrm{ml}$ (Fig. 5). On the other hand, most of the EDE1 and EDE2 mAbs neutralized DC-DENV to $>80 \%$, with some reaching 100\% neutralization (Fig. 5). IB-3, IB-4 and IB-5 mAbs were all poorly neutralizing (Fig. 5). Full binding and neutralization curves for representative $\mathrm{mAbs}$ demonstrated that $\mathrm{mAbs}$ to FLE had diminished binding to DC-DENV, as assessed by ELISA, and failed to fully neutralize infection of DCs with DENV, whereas the neutralization and binding curves for the mAbs to EDE were more closely aligned for C6/36-DENV and DC-DENV (Fig. 6). These data demonstrated the superior performance of $\mathrm{mAbs}$ to EDE in controlling DENV once infection had become established in the mammalian host.

\section{The effect of viral maturation on antibody recognition}

The process of DENV maturation described above means that the DENV glycoprotein shell contains a series of different compositions and conformations depending on the presence of prM in addition to E protein, which is constitutively present in all forms of DENV. To assess a series of different forms of DENV, we compared the binding of mAbs to six preparations of DENV-2. To assess the degree of prM cleavage, we measured the ratio of prM to E protein by ELISA and normalized this to DENV produced in LoVo human colon epithelial cells (LoVo-DENV), cells that lack furin activity and produce almost completely noninfectious immature viral particles with a full complement of prM $^{17}$ (Fig. 7a). The viral preparations were as follows: $\mathrm{C} 6 / 36-\mathrm{DENV}$, which has a relatively high prM content of 54\%; DC-DENV, which has a prM content of 13\%; LoVo-DENV, which has a prM content approaching $100 \%$ (ref. 17); DENV produced in 293T cells overexpressing furin (furin-293T-DENV), which has a prM content of 6\%; DENV produced in native 293T cells, which has a prM content of 63\% (293T-DENV); and DENV incubated at pH 5.5, a condition in which the $\mathrm{E}$ protein irreversibly adopts the trimeric post-fusion conformation $\left(\right.$ acid-DENV) ${ }^{39}$.

EDE1 and EDE2 mAbs did not bind to LoVo-DENV (Fig. 7b), consistent with the observation that at neutral $\mathrm{pH}$, the particles display 60 trimeric spikes and there is no dimer to recapitulate an EDE. The mAbs to FLE showed reduced binding to DENV with a low content of prM; binding curves for DC-DENV and furin-293T-DENV were shifted 1.5 to 2 logs to the right of that for C6/36-DENV. Additionally, binding to LoVo-DENV was even 
more efficient than binding to C6/36-DENV, which emphasized the importance of prM for the exposure and efficient binding of antibodies to the fusion loop ${ }^{32,40}$. These observations indicated that prM, which covers the fusion loop of $\mathrm{E}$ protein at the top of each spike of immature virions, had a weak interaction with E protein, and there was sufficient 'breathing' such that the mAbs to FLE were able to efficiently displace it from the fusion loop. Such 'breathing' was less important in the context of the mature dimers, which displayed reduced binding by the same mAbs. The four IB- $4 \mathrm{mAbs}$, which were isolated from three separate patients, bound most efficiently to acid-DENV but showed negligible neutralization (Figs. 5 and $7 \mathrm{~b}$ ). The mAbs to EDE showed binding to partially processed DENV particles (containing more than 60\% unprocessed prM, such as C6/36-DENV, 293T-DENV or VeroDENV) that was equal to the binding of EDE to nearly fully mature virions, such as DCDENV or furin-293T-DENV (Fig. 7b). The ability of mAbs to EDE to neutralize DENV produced in both insect cells and DCs indicated their ability to bind to particles with varying degrees of maturity containing different amounts of prM.

In a particle that is completely immature, the trimers are totally interlocked, exhibiting more extensive intertrimer contacts than intratrimer contacts ${ }^{16,41}$, consistent with the finding that mAbs to EDE, which required an E dimer to bind, did not react with LoVo-DENV. However, mAbs to EDE reacted as efficiently with particles that had more than $60 \%$ unprocessed prM as they did with totally mature virions of DC-DENV or furin-293T-DENV with exclusively E dimers at their surface (Fig. 7b). At first glance, this might seem odd, as several reports have described the presence of spiky patches of immature lattice (based on $\mathrm{E}$ protein-prM trimers) next to patches of dimers with a smooth appearance on such particles. If these patches were stable, binding of mAbs to EDE should be confined to the mature patch only, and overall binding should be reduced.

However, in contrast to the completely immature particles, the trimeric spikes at the edges of partially mature patches would not be stabilized by three surrounding trimers and would instead be in contact with an adjacent lattice of dimers. EM tomographic reconstruction of hybrid particles containing a smooth area of dimer adjacent to a spiky area of trimer has shown that the boundary between the two, which is about one icosahedral unit in diameter, is not well resolved. It has been proposed that in this area trimer dissociates and dimer is formed, and vice versa ${ }^{16,41}$. Our binding results also indicated that trimers with such a boundary were unstable, and they must exhibit a highly dynamic activity, with their subunits dissociating and reassociating, alternating between dimers and trimers. These data indicate that antibodies to EDE can bind by conformational selection ${ }^{42}$, stabilizing the dimers when they form and displacing prM, which binds at the same site as the mAb to $\mathrm{EDE}^{33}$.

\section{ADE by mAbs to DENV}

ADE of DENV infection is one of the mechanisms postulated to increase the severity of disease upon secondary infection ${ }^{6}$. Antibodies formed in response to the primary infection are proposed to opsonize but not fully neutralize virus and promote Fc receptor-mediated uptake into myeloid cells, driving higher viral loads during secondary infection. ADE can be found at subneutralizing concentrations of almost all antibodies, and its perceived risk complicates strategies for vaccines against DENV. We assessed the ability of the mAbs to 
enhance DENV infection of U937 human histiocytic lymphoma monocytes expressing Fc receptor. All mAbs tested caused ADE, but it was approximately four-to eightfold lower for the EDE group than for the FLE group; the median peak enhancement for the FLE group versus the EDE group was 3,745 to 545 on C6/36-DENV and 2,070 to 480 on DC-DENV (Fig. 8a). These data indicated that mAbs to EDE were less enhancing than mAbs to the fusion loop and that the performance of the mAbs to EDE would be best illustrated by the reporting of neutralization as a $90 \%$ neutralization titer $\left(\mathrm{NT}_{90}\right)$ (Fig. $8 \mathrm{~b}$ ).

\section{DISCUSSION}

Our analysis here of the human antibody response after natural DENV infection has identified a new epitope for mAbs to DENV that was highly conserved and cross-reacted across virus serotypes with neutralization titers in the low picomolar range. EDE1 and EDE2 mAbs efficiently neutralized virus produced in both insect cells and primary human cells; these results highlight a previously described weakness of classic FLE-specific mAbs. mAbs to FLE, although highly serotype cross-reactive, were unable to fully neutralize virus produced in primary human cells. Antibodies to EDE have been shown to bind to the two polypeptide subunits of the E protein organized as the basic head-to-tail dimers of the mature virion, with the glycan chains modulating the interaction ${ }^{33}$.

The immunodominance of recognition in a patient was unexpected. As the epitopes overlap, it is possible that the most avid antibody might outcompete other antibodies and undergo affinity maturation and hence dominate the response, which would lead to a stochastic 'choice' between FLE and EDE. However, the responses to EDE or FLE were polyclonal in patients (with different recombinations of variable, diversity and joining regions), which would make this less likely as an explanation. The production of cross-reactive antibodies to EDE in the single primary case was interesting, and further studies should determine how common the production of antibodies to EDE is during primary infection and also the degree and persistence of the EDE response in serum following convalescence.

The differences in the neutralization of DC-produced DENV versus that of insect cellproduced DENV resulted from differences in the prM content of the DENV particles. Both insect cell lines and tumor cell lines (such as Vero) produced virus with higher prM content than that of virus produced in primary DCs. Our results have highlighted a dynamic activity of the $\mathrm{E}$ protein on virions, showing in particular that on partially mature particles there was continuous 'breathing' between dimers and trimers, because otherwise the antibodies to EDE would not bind equally to partially mature viral particles and fully mature viral particles.

The relatively high content of prM in DENV produced in insect or Vero cells, which is commonly used to determine neutralization titers, might overestimate neutralization potential for the vast bulk of virus produced by iterative replication cycles in the infected host with a low prM content. This tendency to misestimate could be compounded further by the use of $\mathrm{NT}_{50}$ values to assess responses, which overestimate the efficacy of the anti-FLE component of the response. $\mathrm{NT}_{90}$ values might therefore better represent protection. Furthermore, full delineation of the correlates of protection to DENV infection is an 
imperative following the results of the Sanofi Pasteur dengue vaccine trial ${ }^{8}$. We propose that the induction of antibodies targeting this cross-reactive EDE would be an important feature of any successful vaccine against DENV. Assays that measure the neutralization of DENV produced in primary human cells or cells overexpressing furin, together with a measure of different components of the response (for example, E protein versus prM, and FLE versus EDE) should be developed to design more robust and specific assays that can be used to predict true immunological protection after immunization.

Finally, current vaccine strategies use a tetravalent formulation with the aim of raising a balanced type-specific response to all four DENV serotypes. The description here of such potent and cross-reactive antibodies indicates a way for the development of subunit vaccines containing the desired epitope and possibly heterologous prime-boost strategies to recapitulate responses seen in natural sequential infections. The discovery of the EDE might make the generation of potent and broadly neutralizing antibodies a realistic goal for future vaccines and might even allow the possibility of a single universal immunogen.

\section{ONLINE METHODS}

\section{Samples}

Blood samples were collected from inpatients after written informed consent. The study protocol was approved by the Scientific and Ethical Committee of the Hospital for Tropical Diseases, the Oxford Tropical Research Ethical Committee and the Riverside Ethics Committee in the UK. Laboratory confirmation of DENV infection was determined by RTPCR detection of DENV nucleic acid (which also confirmed the infecting serotype), NS1 antigen lateral flow test or seroconversion in an ELISA of IgM. Disease severity was classified according to 1997 World Health Organization criteria. Of the patients enrolled in the study, two patients were classified as mildly symptomatic of dengue fever, and five patients were classified as severely symptomatic of dengue hemorrhagic fever, with plasma leakage and bleeding (Supplementary Table 1). Secondary infection was defined based on the ratio of DENV-specific IgM to IgG $<1.2$ on or after day 6 of illness ${ }^{43}$. Blood samples for B cell sorting were collected during the hospitalization period at time points when the blood plasmablast population was apparent. Peripheral blood mononuclear cells were isolated from whole blood by Ficoll-Hypaque density-gradient centrifugation and resuspended in $10 \%$ FCS in RPMI medium, for immediate use.

\section{Cells, reagents and antibodies}

The C6/36 cell line (a gift from AFRIMS), derived from the mosquito Aedes albopictus, was cultured in Leibovitz L-15 at $28^{\circ} \mathrm{C}$. Vero cells (a gift from AFRIMS), U937 cells, and 293T cells or $293 \mathrm{~T}$ cells transfected to express furin were grown at $37^{\circ} \mathrm{C}$ in MEM, RPMI-1640 medium, and DMEM, respectively. All media were supplemented with $10 \%$ heat-inactivated fetal bovine serum, 100 units $/ \mathrm{ml}$ penicillin, $100 \mu \mathrm{g} / \mathrm{ml}$ streptomycin and $2 \mathrm{mM} \mathrm{L}$-glutamine. The furin-deficient LoVo cell line (CCL-229; ATCC) was maintained in F-12 medium as recommended. Monocyte-derived DCs were prepared as described ${ }^{44}$. 
Peridinin chlorophyll protein-conjugated anti-human CD3 (345766), peridinin chlorophyll protein-conjugated anti-human CD20 (345794), fluorescein isothiocyanate-conjugated antihuman CD27 (555440) and allophycocyanin-conjugated anti-human CD38 (555462) were from BD Pharmingen. Phycoerythrin-conjugated anti-human CD19 (R0808), horseradish peroxidase-conjugated anti-human $\mathrm{IgG}$ (P0214) and peroxidase-conjugated anti-mouse Ig (P0447) were from Dako. Alkaline phosphatase (ALP)-conjugated anti-human IgG (A9544), biotin-conjugated anti-human IgG (B1140), Biotin-conjugated anti-human IgM (B1265) and ALP-streptavidin (S2890) were from Sigma. Mouse monoclonal anti-DENV E (4G2) and anti-DENV prM (1H10) were gifts from AFRIMS, C. Puttikhunt and W. Kasinrerk ${ }^{45}$. AntiDENV NS3 (E1D8) was a gift from E. Harris.

Restriction enzymes, Agel-HF (R3552), SalI-HF (R3138), XhoI (R0146), BsiWI (R0553), calf-intestinal ALP (M0290) and T4 DNA ligase (M0202L) were from NEB. Bovine serum albumin (BSA, A7030), p-nitrophenylphosphate (PNPP, N2770-50), RPMI1640 (R8758), DMEM (D5046) and diaminobenzidine (D5905) were from Sigma. MEM (31095) was from Gibco.

\section{Viral stocks}

DENV-1 (Hawaii), DENV-2 (16681), DENV-3 (H87) and DENV-4 (H241) (gifts from AFRIMS) were grown in C6/36 cells. All of these virus preparations contained substantial amounts of partially mature particles, as indicated by their prM content. In addition, DENV-2 was propagated in DCs, LoVo cells, 293T cells and 293T cells transfected to express furin, and cell-free supernatants were collected and stored at $-80{ }^{\circ} \mathrm{C}$. Viral titers were determined by a focus-forming assay on Vero cells and results are presented as focusforming units per $\mathrm{ml}$ (ref. 46).

\section{Generation of DENV-specific human mAbs}

DENV-specific human mAbs were generated from activated B cells or plasmablasts ${ }^{20,21}$. Perimpheral blood mononuclear cells were stained with anti-CD3 (345766; BD Pharmingen), anti-CD19 (R0808; Dako), anti-CD20 (345794; BD Pharmingen), anti-CD27 (555440; BD Pharmingen) and anti-CD38 (555462; BD Pharmingen). Activated antibodysecreting cells were then gated as $\mathrm{CD} 19^{+}, \mathrm{CD} 3^{-}, \mathrm{CD} 20^{\text {lo }}$ to $\mathrm{CD} 20^{-}, \mathrm{CD} 27^{\mathrm{hi}}$ and $\mathrm{CD} 38^{\mathrm{hi}}$. A single antibody-secreting cell was sorted into each well of 96-well PCR plates containing RNase inhibitor (N2611; Promega). Plates were centrifuged briefly and frozen on dry ice before storage at $-80^{\circ} \mathrm{C}$. RT-PCR (210212; Qiagen) and nested PCR (203205; Qiagen) were then performed to amplify genes encoding $\gamma$-chain, $\lambda$-chain and $\kappa$-chain with 'cocktails' of primers specific for IgG. Products of PCR analyzing genes encoding heavy and light chains were then digested with the appropriate restriction endonuclease(s) and were cloned into expression vectors for IgG1 or immunoglobulin $\kappa$-chain or $\lambda$-chain (gifts from $\mathrm{H}$. Wardemann). For the expression of antibodies, plasmids enoding heavy and light chains were cotransfected into the 293T cell line by the polyethylenimine method (408727; Sigma), and antibody-containing supernatants were harvested for further characterization. 


\section{Enzyme-linked immunospot assay}

Enzyme-linked immunospot plates (MSIPN4510; Millipore) were coated with either antibody to human immunoglobulin (H17000; Caltag) or ultraviolet irradiation-inactivated DENV-1-DENV-4. Plates were washed with RPMI medium and nonspecific binding was blocked by incubation for $1 \mathrm{~h}$ with $1 \%$ BSA in RPMI medium. Sorted antibody-secreting cells were added at 500 cells in wells coated with anti-immunoglobulin and DENV and samples were incubated overnight at $37{ }^{\circ} \mathrm{C}$ in $5 \% \mathrm{CO}_{2}$. Plates were washed and then were incubated for $2 \mathrm{~h}$ at room temperature with biotinylated antibody to human $\operatorname{IgG}$ (B1140; Sigma) or IgM (B1265; Sigma), followed by streptavidin-ALP (S2890; Sigma). Reactions were developed and spots were counted with an AID Elispot plate reader.

\section{Detection of DENV specificity and serotype cross-reactivity by ELISA}

Supernatants of mock-infected (uninfected) cells and cells infected with DENV-1-DENV-4 were captured separately onto a MAXISORP immuno-plate (442404; NUNC) coated with mouse antibody to E protein (4G2). Wells in which DENV was captured were then incubated with $1 \mu \mathrm{g} / \mathrm{ml}$ of human mAbs (from our patients), followed by ALP-conjugated antibody to human IgG (A9544; Sigma). Reactions were visualized by the addition of PNPP substrate and were stopped with $\mathrm{NaOH}$. The absorbance was measured at $405 \mathrm{~nm}$.

\section{ELISA of recombinant soluble DENV E protein}

Plates were directly coated with $150 \mathrm{ng}$ recombinant soluble E protein (a gift from A Flanagan), and bovine serum albumin (BSA) was used as negative control antigen. Proteincoated wells were then incubated with $1 \mu \mathrm{g} / \mathrm{ml}$ of human mAbs (from our patients), followed by ALP-conjugated antibody to human IgG (A9544; Sigma). PNPP substrate was finally added and the absorbance was measured at $405 \mathrm{nM}$.

\section{Immunoblot analysis}

For the analysis, DENV-containing supernatants from C6/36 cells were prepared in unheated and nonreducing conditions and were separated by electrophoresis through $12 \%$ SDS polyacryramide gels and transferred by electroblot onto nitrocellulose membranes (RPN 303E; Amersham). Nonspecific binding was then blocked with 5\% skimmed milk and the membranes were probed with DENV-specific human mAbs (from our patients) followed by horseradish peroxidase-conjugated antibody to human IgG (P0214; Sigma). Membranes were developed with enhanced chemiluminescence substrate (RPN2106; Amersham).

\section{Antibody epitope mapping with VLP mutants}

Full-length prM/E of DENV-1 was cloned into the expression vector pHLsec to generate VLP (constructed by A. Flanagan) ${ }^{47}$. VLP mutants were generated by PCR-based sitedirected mutagenesis ${ }^{48}$. Mutagenic PCR was performed to substitute selected amino acid residues in the E protein with alanine through the use of Pfx DNA polymerase (11708021; Invitrogen); if the amino acid was already alanine, it was replaced with glycine. After treatment with DpnI (R01765; NEB), PCR products were transformed into Escherichia coli strain DH5a. All mutations were confirmed by sequencing (Macrogen). Plasmids were transfected into the $293 \mathrm{~T}$ cell lines by the polyethylenimine method and culture supernatants 
were harvested for epitope mapping. Not all changes to alanine within the antibody footprint will block binding, as the change may be too conservative, the interaction is not strong enough or the interaction with a given amino acid may rely on conserved main-chain interaction and not with the mutant side chain.

The prM content of each VLP preparation, assessed by the ratio of prM to E protein, was representative of partially mature particles similar to infectious virus produced in the 293T cell line. To identify epitope-specific antibodies, wild-type and mutant VLPs were captured with mouse anti-prM (1H10; a gift from C. Puttikhunt). DENV-specific human antibody to E protein (from our patients) was then added at $1-5 \mu \mathrm{g} / \mathrm{ml}$, followed by ALP-conjugated antibody to human IgG (A9544; Sigma). Finally, PNPP substrate was added, the reaction stopped by the addition of $\mathrm{NaOH}$ and absorbance was measured at $405 \mathrm{~nm}$. The relative recognition index was calculated as follows: [absorbance of mutant VLP/absorbance of wild-type VLP] (recognized by the test $\mathrm{mAb}$ )/[absorbance of mutant VLP/absorbance of wild-type VLP] (recognized by a group of four mixed mAbs: 751 C5 (FLE), 751 C10 (nonFLE), 749(2) A7 (non-FLE) and 753(3) B11 (non-FLE)).

\section{Neutralization and enhancement assays}

The neutralization potential of mAbs was determined by the focus reduction neutralization test (FRNT), whereby the reduction in the number of infected foci is compared with that in the control condition (no antibody). Serially diluted antibody was mixed with virus and was incubated for $1 \mathrm{~h}$ at $37^{\circ} \mathrm{C}$. The mixtures were then transferred to Vero cells and were incubated for $3 \mathrm{~d}$. The focus-forming assay was then carried out with mAb 4G2 to E protein, followed by goat anti-mouse immunoglobulin (P0047; Sigma), conjugated to horseradish peroxidase. The reaction was visualized by the addition of DAB substrate. The percentage of focus reduction was calculated for each antibody dilution. 50\% FRNT values were determined from graphs of percentage reduction versus concentration of antibodies with the probit program from the SPSS package.

For the ADE assay, serially diluted antibody was preincubated with virus for $1 \mathrm{~h}$ at $37^{\circ} \mathrm{C}$ and then transferred to U937 cells (Fc receptor-bearing human monocyte cell lines), followed by incubation for $4 \mathrm{~d}$. Supernatants were harvested and were titrated on Vero cells by a focus-forming assay. The titers of virus were presented as focus-forming units per $\mathrm{ml}$ and the 'increment' was calculated by comparison to viral titers in the absence of antibody.

\section{DENV-binding ELISA}

To determine the binding affinity of antibody to DENV generated from various cell types, supernatants of mock-infected cells (no virus) or DENV-2 produced from C6/36 cells, DCs, 293T cells, 293T cells transfected to express furin or LoVo cells were captured onto plates coated with mAb 4G2 and then were incubated with serial dilutions of DENV-specific human mAb (from our patients) followed by ALP-conjugated anti-human IgG (A9544; Sigma). Reactions were developed by the addition of PNPP substrate and were stopped by the addition of $\mathrm{NaOH}$. The absorbance was measured at $405 \mathrm{~nm}$. Antigen loading of the various viral forms and interday variation between experiments in absorbance readings were 
normalized by a control ELISA with a humanized version of $\mathrm{mAb} 3 \mathrm{H} 5$, which is specific to domain III of DENV-2.

The ratio of prM to E protein was measured by capture of virus onto plates coated with $\mathrm{mAb}$ 4G2, and DENV-2 E protein was measured by ELISA with mAb 3H5 (specific for DENV-2 domain III). The ratio was calculated as absorbance for prM / absorbance for E protein.

Percent cleavage of prM was then calculated with reference to virus produced in LoVo cells, which was assumed to be $100 \%$ uncleaved.

\section{Acid-treated virus}

The method has been described ${ }^{49}$. Culture medium from DENV-2-infected C6/36 cells was precipitated with PEG, and viral pellets were resuspended with TNE buffer (12 mM Tris, $120 \mathrm{mM} \mathrm{NaCl}$ and $1 \mathrm{mM}$ EDTA, $\mathrm{pH}$ 8.0). Virus was then acidified by the addition of a buffer of $0.05 \mathrm{M}$ MES and $0.1 \mathrm{M} \mathrm{NaCl}$ to achieve a $\mathrm{pH}$ of 5.5. After incubation for $10 \mathrm{~min}$ at $37^{\circ} \mathrm{C}$, the virus was adjusted back to a pH of 8.0 by the addition of a buffer of $0.1 \mathrm{M}$ diethanolamine and $0.1 \mathrm{M} \mathrm{NaCl}$.

\section{Cryo-EM reconstruction}

Virus preparation- $\mathrm{C} 6 / 36$ cells were cultured at $32{ }^{\circ} \mathrm{C}$ in the presence of $5 \% \mathrm{CO}_{2}$. During cell passaging, we scraped cells from the dish, avoiding exposure of cells to trypsin. Thirty-five Corning tissue-culture treated culture dishes $(\mathrm{D} \times \mathrm{H}, 150 \mathrm{~mm} \times 25 \mathrm{~mm})$, each containing C6/36 cells in $30 \mathrm{ml}$ of medium, were infected with DENV-2, New Guinean strain. Four days after infection, cell culture medium was collected and was centrifuged for $30 \mathrm{~min}$ in a Beckmann centrifuge $(11,000 \mathrm{~g})$ to pellet large debris to be discarded. The supernatant harvested was centrifuged for $1 \mathrm{~h}$ in $26 \times 38.5 \mathrm{ml}$ centrifuge tubes in a Beckmann centrifuge $(141,000 \mathrm{~g})$ to collect the virus-containing pellet. The sample was left for $2 \mathrm{~h}$ at $4{ }^{\circ} \mathrm{C}$ and subsequently the centrifuged virus was resuspended in PBS buffer by soaking of the pellet in the buffer for $10 \mathrm{~min}$. The resuspended sample was then loaded at the top of a sucrose gradient (15\% to 50\%) and was centrifuged for $2 \mathrm{~h}$ at $130,000 \mathrm{~g}$ (Beckman Coulter SW41) at $4{ }^{\circ} \mathrm{C}$. A band was located at about one-third the distance from the top of the gradient. The gradient material above the band was removed with a pipette; then, the virus-containing band was carefully collected with another pipette, or the band was directly collected via a syringe. The collected viral sample $(1 \mathrm{ml})$ was diluted to a volume of $\sim 12 \mathrm{ml}$ with PBS buffer and an Amicon Ultra filter was used to concentrate the sample. The resulting $50 \mu \mathrm{l}$ of purified virus was ready for cryo-EM.

The Fab fragment of mAb 747(4)B7 was purified by size-exclusion chromatography and was concentrated to $2.5 \mathrm{mg} / \mathrm{ml}$ in Tris- $\mathrm{NaCl}$ buffer $(50 \mathrm{mM}$ Tris, $\mathrm{pH} 8$, and $0.5 \mathrm{M} \mathrm{NaCl})$ with Vivaspin protein concentrators (10 kDa cutoff).

Electron microscopy-Purified virus was mixed with FabB7 (1:4, vol/vol), aiming to reach molecular excess of the Fab. Aliquots of $3 \mu \mathrm{l}$ of the mixture were placed on glowdischarged holey carbon grids (Quantifoil Cu R2/2). Grids were blotted for $5 \mathrm{~s}$ and were flash frozen in liquid ethane with an FEI Vitrobot Mark IV. Grids were transferred to an FEI Titan Krios electron microscope operating at $300 \mathrm{kV}$. Images were recorded by Leginon 
with a Gatan K2 Summit detector in counting mode on the Titan Krios microscope at a nominal magnification of $\times 29,000$ (which yields a pixel size of $1.28 \AA$ ). Underfocus values in the final $\mathrm{K} 2$ data set ranged from $0.2 \mu \mathrm{m}$ to $3.5 \mu \mathrm{m}$, and 25 frames of each movie were used for later image processing.

Image processing-We manually selected more than 8,000 particles with a box size of 640 pixels from 1,056 micrographs in the final $\mathrm{K} 2$ data set. Contrast transfer function parameters were estimated with CTFFIND3 program for finding contrast transfer functions in electron micrographs ${ }^{50}$. We filtered the published DENV-2 cryo-EM map $\left({ }^{20}\right.$ as a starting model according to online instructions for the RELION ('regularized likelihood optimization') computer program for refinement of cryo-EM data ${ }^{51}$, then we used RELION for two-dimensional classification and 'gold-standard' Fourier shell correlation-based threedimensional auto refine. To boost the computation, we 'down-sampled' the original particle images to 320 pixels, which resulted in a pixel size of $2.56 \AA$. The final resolution of the map is $10.24 \AA$ when a cutoff of 0.5 is used and $8.5 \AA$ when a cutoff of 0.143 is used (Supplementary Fig. 3).

Cryo-EM map interpretation-The $3.5 \AA$ cryo-EM of the DENV-2 viral particle (EMDB accession code, 5520) was used to interpret the E/M glycoprotein shell of the reconstruction of the DENV-2-Fab 747(4)B7 complex. Because the two maps are in the same reference frame and have the same icosahedral orientation, they superpose directly. The two maps were normalized, and the densities above $4 \sigma$ were used for correlation optimization. The correlation coefficient, obtained with the program Chimera, was 0.98. An attempt to optimize the position and orientation of the particle led to a shift of less than 1 pixel and $0^{\circ}$ rotation, which indicated that the particle was already at its correlation maximum. Density maps were presented with Chimera.

\section{Supplementary Material}

Refer to Web version on PubMed Central for supplementary material.

\section{ACKNOWLEDGMENTS}

\footnotetext{
We thank the Armed Forces Research Institute of Medical Sciences of Thailand (AFRIMS), C. Puttikhunt (National Center for Genetic Engineering and Biotechnology, Thailand) and W. Kasinrerk (Chiang Mai University) for the mouse mAb 4G2 to DENV E protein and mAb 1H10 to DENV; E. Harris (University of California Berkeley School of Public Health) for mAb E1D8 to DENV NS3 protein; H. Wardemann (Max Planck Institute for Infection Biology) for expression vectors for IgG1 or immunoglobulin $\kappa$-chain or $\lambda$-chain; A Flanagan (University of Oxford) for recombinant soluble E protein; the staff at Oxford University Clinical Research Unit Viet Nam for sample collection; and N. Ferguson (Imperial College London) for statistical advice. We acknowledge the use of instruments at the Electron Imaging Center for Nanomachines, supported by University of California Los Angeles and the US National Institutes of Health (1S10OD018111 and NSF DBI-1338135). Supported by the Medical Research Council UK, the Wellcome Trust (G.R.S.), the National Institutes for Health Research Biomedical Research Centre, the US National Institutes of Health (GM071940 and AI094386), European Commission Seventh Framework Programme (FP7/2007-2013; DENFREE project, 282 378) and the Thailand Research Fund through the Royal Golden Jubilee Ph.D. Program (S.S. and J.M.).
}

\section{References}

1. Westaway, EG.; Blok, J. Dengue and Dengue Hemorrhagic fever. Gubler, DJ.; Kuno, G., editors. CABI Publishing; Oxford, UK: 1997. p. 147-173. 
2. Bhatt S, et al. The global distribution and burden of dengue. Nature. 2013; 496:504, 507. [PubMed: 23563266]

3. Mongkolsapaya $\mathbf{J}$, et al. Original antigenic sin and apoptosis in the pathogenesis of dengue hemorrhagic fever. Nat. Med. 2003; 9:921-927. [PubMed: 12808447]

4. Mongkolsapaya J, et al. T cell responses in dengue hemorrhagic fever: are cross-reactive $\mathrm{T}$ cells suboptimal? J. Immunol. 2006; 176:3821-3829. [PubMed: 16517753]

5. Sangkawibha N, et al. Risk factors in dengue shock syndrome: a prospective epidemiologic study in Rayong, Thailand. I. The 1980 outbreak. Am. J. Epidemiol. 1984; 120:653-669. [PubMed: 6496446]

6. Halstead SB. Neutralization and antibody-dependent enhancement of dengue viruses. Adv. Virus Res. 2003; 60:421-467. [PubMed: 14689700]

7. Murphy BR, Whitehead SS. Immune response to dengue virus and prospects for a vaccine. Annu. Rev. Immunol. 2011; 29:587-619. [PubMed: 21219187]

8. Sabchareon A, et al. Protective efficacy of the recombinant, live-attenuated, CYD tetravalent dengue vaccine in Thai schoolchildren: a randomised, controlled phase $2 b$ trial. Lancet. 2012; 380:1559_ 1567. [PubMed: 22975340]

9. Capeding MR, et al. Clinical efficacy and safety of a novel tetravalent dengue vaccine in healthy children in Asia: a phase 3, randomised, observer-masked, placebo-controlled trial. Lancet. 2014; 384:1358-1365. [PubMed: 25018116]

10. Kuhn RJ, et al. Structure of dengue virus: implications for flavivirus organization, maturation, and fusion. Cell. 2002; 108:717-725. [PubMed: 11893341]

11. Mukhopadhyay S, Kuhn RJ, Rossmann MG. A structural perspective of the flavivirus life cycle. Nat. Rev. Microbiol. 2005; 3:13-22. [PubMed: 15608696]

12. Li L, et al. The flavivirus precursor membrane-envelope protein complex: structure and maturation. Science. 2008; 319:1830-1834. [PubMed: 18369147]

13. Yu IM, et al. Structure of the immature dengue virus at low $\mathrm{pH}$ primes proteolytic maturation. Science. 2008; 319:1834-1837. [PubMed: 18369148]

14. Bressanelli S, et al. Structure of a flavivirus envelope glycoprotein in its low-pH-induced membrane fusion conformation. EMBO J. 2004; 23:728-738. [PubMed: 14963486]

15. Modis Y, Ogata S, Clements D, Harrison SC. Structure of the dengue virus envelope protein after membrane fusion. Nature. 2004; 427:313-319. [PubMed: 14737159]

16. Plevka P, et al. Maturation of flaviviruses starts from one or more icosahedrally independent nucleation centres. EMBO Rep. 2011; 12:602-606. [PubMed: 21566648]

17. Dejnirattisai $\mathrm{W}$, et al. Cross-reacting antibodies enhance dengue virus infection in humans. Science. 2010; 328:745-748. [PubMed: 20448183]

18. Fibriansah G, et al. Structural changes in dengue virus when exposed to a temperature of 37C. J. Virol. 2013; 87:7585-7592. [PubMed: 23637405]

19. Zhang X, et al. Dengue structure differs at the temperatures of its human and mosquito hosts. Proc. Natl. Acad. Sci. USA. 2013; 110:6795-6799. [PubMed: 23569243]

20. Smith K, et al. Rapid generation of fully human monoclonal antibodies specific to a vaccinating antigen. Nat. Protoc. 2009; 4:372-384. [PubMed: 19247287]

21. Tiller T, et al. Efficient generation of monoclonal antibodies from single human B cells by single cell RT-PCR and expression vector cloning. J. Immunol. Methods. 2008; 329:112-124. [PubMed: 17996249]

22. Balakrishnan T, et al. Dengue virus activates polyreactive, natural IgG B cells after primary and secondary infection. PLoS ONE. 2011; 6:e29430. [PubMed: 22216280]

23. Wrammert J, et al. Rapid and massive virus-specific plasmablast responses during acute dengue virus infection in humans. J. Virol. 2012; 86:2911-2918. [PubMed: 22238318]

24. Zhang X, et al. Cryo-EM structure of the mature dengue virus at 3.5-A resolution. Nat. Struct. Mol. Biol. 2013; 20:105-110. [PubMed: 23241927]

25. Modis Y, Ogata S, Clements D, Harrison SC. A ligand-binding pocket in the dengue virus envelope glycoprotein. Proc. Natl. Acad. Sci. USA. 2003; 100:6986-6991. [PubMed: 12759475] 
26. Ferlenghi I, et al. Molecular organization of a recombinant subviral particle from tick-borne encephalitis virus. Mol. Cell. 2001; 7:593-602. [PubMed: 11463384]

27. Beltramello M, et al. The human immune response to dengue virus is dominated by highly crossreactive antibodies endowed with neutralizing and enhancing activity. Cell Host Microbe. 2010; 8:271-283. [PubMed: 20833378]

28. Lai C-Y, et al. Antibodies to envelope glycoprotein of dengue virus during the natural course of infection are predominantly cross-reactive and recognize epitopes containing highly conserved residues at the fusion loop of domain II. J. Virol. 2008; 82:6631-6643. [PubMed: 18448542]

29. Costin JM, et al. Mechanistic study of broadly neutralizing human monoclonal antibodies against dengue virus that target the fusion loop. J. Virol. 2013; 87:52-66. [PubMed: 23077306]

30. Smith SA, et al. The potent and broadly neutralizing human dengue virus-specific monoclonal antibody 1C19 reveals a unique cross-reactive epitope on the bc loop of domain II of the envelope protein. mBio. 2013; 4:e00873-00813. [PubMed: 24255124]

31. Tsai WY, et al. High-avidity and potently neutralizing cross-reactive human monoclonal antibodies derived from secondary dengue virus infection. J. Virol. 2013; 87:12562-12575. [PubMed: 24027331]

32. Cherrier MV, et al. Structural basis for the preferential recognition of immature flaviviruses by a fusion-loop antibody. EMBO J. 2009; 28:3269-3276. [PubMed: 19713934]

33. Rouvinski A, et al. Recognition determinants of broadly neutralizing human antibodies against dengue viruses. Nature. in the press.

34. Kaufmann B, et al. Neutralization of West Nile virus by cross-linking of its surface proteins with Fab fragments of the human monoclonal antibody CR4354. Proc. Natl. Acad. Sci. USA. 2010; 107:18950-18955. [PubMed: 20956322]

35. Teoh EP, et al. The structural basis for serotype-specific neutralization of dengue virus by a human antibody. Sci. Transl. Med. 2012; 4:139ra183.

36. de Alwis R, et al. Identification of human neutralizing antibodies that bind to complex epitopes on dengue virions. Proc. Natl. Acad. Sci. USA. 2012; 109:7439-7444. [PubMed: 22499787]

37. Fibriansah G, et al. A potent anti-dengue human antibody preferentially recognizes the conformation of E protein monomers assembled on the virus surface. EMBO Mol. Med. 2014; 6:358-371. [PubMed: 24421336]

38. Wu SJ, et al. Human skin Langerhans cells are targets of dengue virus infection. Nat. Med. 2000; 6:816-820. [PubMed: 10888933]

39. Allison SL, et al. Oligomeric rearrangement of tick-borne encephalitis virus envelope proteins induced by an acidic pH. J. Virol. 69:695-700. [PubMed: 7529335]

40. Nelson S, et al. Maturation of West Nile virus modulates sensitivity to antibody-mediated neutralization. PLoS Pathog. 2008; 4:e1000060. [PubMed: 18464894]

41. Plevka P, Battisti AJ, Sheng J, Rossmann MG. Mechanism for maturation-related reorganization of flavivirus glycoproteins. J. Struct. Biol. 2014; 185:27-31. [PubMed: 24252771]

42. Weikl TR, Paul F. Conformational selection in protein binding and function. Protein Sci. 2014; 23:1508-1518. [PubMed: 25155241]

43. Trung DT, et al. Clinical features of dengue in a large Vietnamese cohort: intrinsically lower platelet counts and greater risk for bleeding in adults than children. PLoS Negl. Trop. Dis. 2012; 6:e1679. [PubMed: 22745839]

44. Dejnirattisai W, et al. A complex interplay among virus, dendritic cells, T cells, and cytokines in dengue virus infections. J. Immunol. 2008; 181:5865-5874. [PubMed: 18941175]

45. Puttikhunt $\mathrm{C}$, et al. Novel anti-dengue monoclonal antibody recognizing conformational structure of the prM-E heterodimeric complex of dengue virus. J. Med. Virol. 2008; 80:125-133. [PubMed: 18041028]

46. Sittisombut N, et al. Lack of augmenting effect of interferon- $\gamma$ on dengue virus multiplication in human peripheral blood monocytes. J. Med. Virol. 1995; 45:43-49. [PubMed: 7536230]

47. Aricescu AR, Lu W, Jones EY. A time- and cost-efficient system for high-level protein production in mammalian cells. Acta Crystallogr. D Biol. Crystallogr. 2006; 62:1243-1250. [PubMed: 17001101] 
48. Zheng L, Baumann U, Reymond JL. An efficient one-step site-directed and site-saturation mutagenesis protocol. Nucleic Acids Res. 2004; 32:e115. [PubMed: 15304544]

49. Heinz FX, et al. Structural changes and functional control of the tick-borne encephalitis virus glycoprotein E by the heterodimeric association with protein prM. Virology. 1994; 198:109-117. [PubMed: 8259646]

50. Mindell JA, Grigorieff N. Accurate determination of local defocus and specimen tilt in electron microscopy. J. Struct. Biol. 2003; 142:334-347. [PubMed: 12781660]

51. Scheres SHW. RELION: Implementation of a Bayesian approach to cryo-EM structure determination. J. Struct. Biol. 2012; 180:519-530. [PubMed: 23000701] 
a DENV serotype specificity

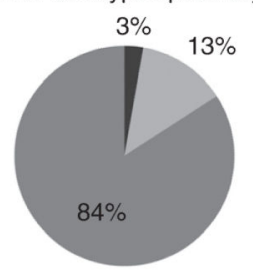

b

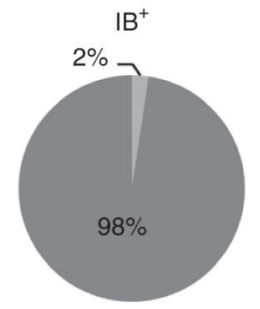

- Serotype specific Partially cross-reactive - Fully cross-reactive

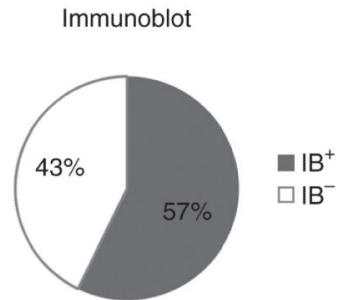

Figure 1.

Characterization of human mAbs to DENV. (a) Serotype specificity, assessed by ELISA, and reaction of $145 \mathrm{mAbs}$ to DENV E protein, assessed by immunoblot analysis. (b) Serotype specificity of $\mathrm{IB}^{+}$and $\mathrm{IB}^{-} \mathrm{mAbs}$. Data are representative of two experiments. 

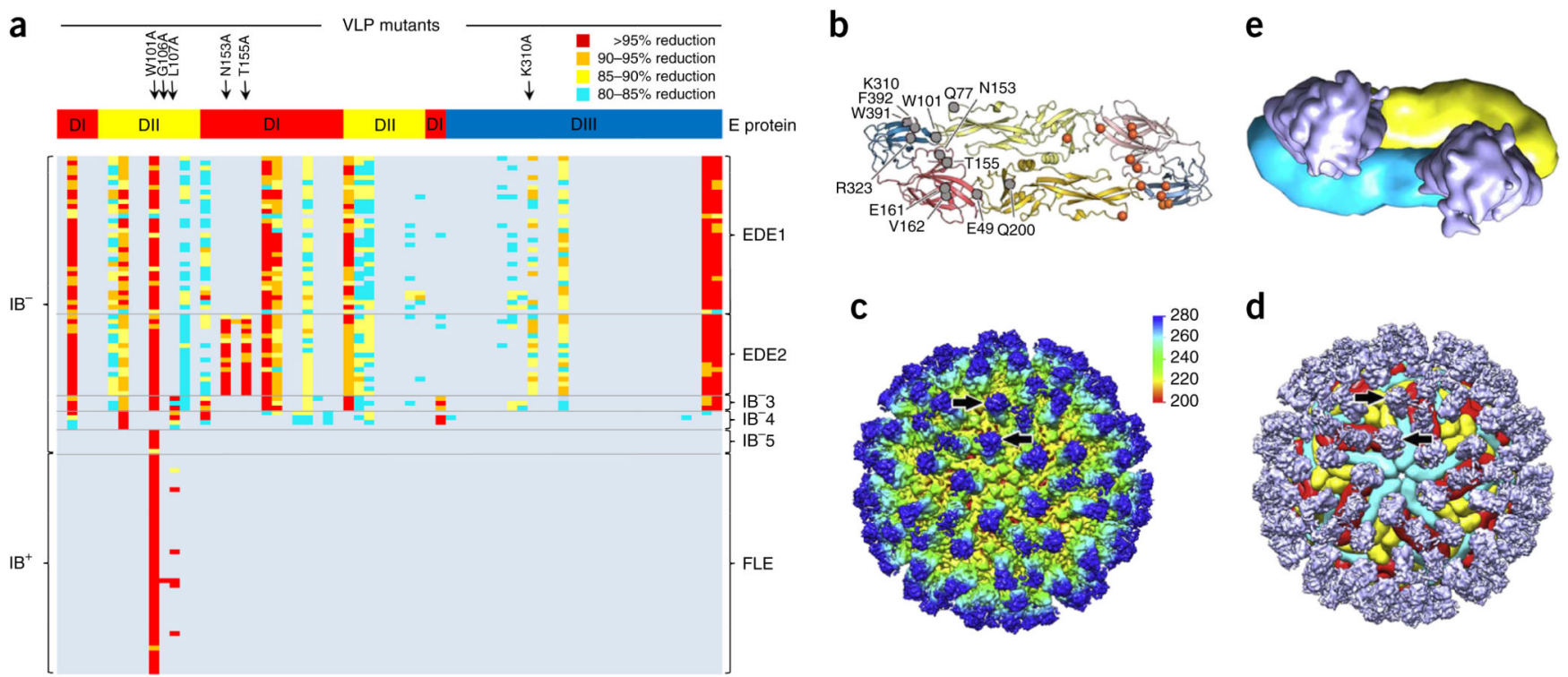

Figure 2.

Epitope mapping of anti-DENV. (a) Epitope mapping with a panel of mutant VLPs (full results, Supplementary Table 2). Top, positions of substitutions in the domain structure of DENV E protein (horizontal stripe at top: DI, domain I; DII, domain II; DIII, domain III), including substitutions marking the fusion loop around Trp101 and disrupting the Asn153 glycosylation motif. Right and left margins, antibody groups (gray horizontal lines demarcate groups). (b) Positions of substitutions leading to $>90 \%$ lower binding, mapped to either end of the E dimer (gray and orange circles). This model was based on the DENV-2 E dimer structure (PDB accession code 1OAN). (c) Three-dimensional reconstruction of the DENV-2 particle in complex with Fab 747(4)B7, calculated to a resolution beyond $10 \AA$ (Supplementary Fig. 3). The contour level shown corresponds to $2 *$ sigma (the root mean square deviation of grid values in the map). The reconstruction is colored to provide radial depth (red (inner radii) to yellow green to cyan to blue (outer radii) (key)). Here, the projecting constant domain of the Fab is dark blue, the variable domain is cyan-green, the $\mathrm{E}$ protein shell is green-yellow and internal features (parts of the membrane) are seen in red through holes in the glycoprotein shell. Arrows point to the density of Fab 747(4)B7 bound to the same E dimer. (d) The density corresponding to Fab 747(4)B7 complex superimposed on the $3.5 \AA$ cryo-EM reconstruction of the DENV-2 virion ${ }^{24}$, after subtraction of the E shell from the reconstruction of the complex. The three independent $\mathrm{E}$ and $\mathrm{M}$ proteins in the icosahedral asymmetric unit are in red for the subunit adjacent to the icosahedral twofold axes, are in yellow for that about the threefold axes, and are in cyan for that about the fivefold axis. Arrows point to the same density as in c. (e) A single E dimer showing Fab binding at the dimer interface matching the cluster of residues sensitive to binding, as determined by alanine scanning on DENV-2 VLPs in $\mathbf{b}$. 
747

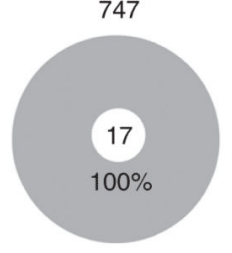

752

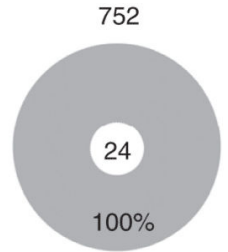

749
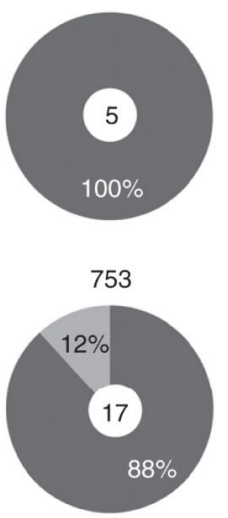

750

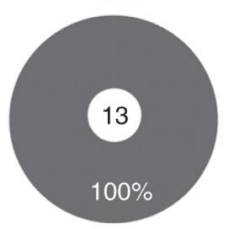

758

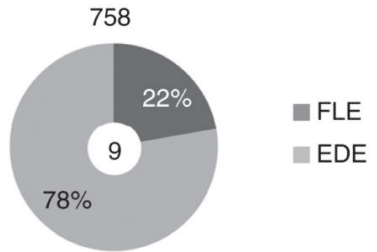

Figure 3.

$\mathrm{mAbs}$ to FLE versus $\mathrm{mAbs}$ to EDE in individual patients. Distribution of the responses to FLE and EDE (as percentages) by seven patients infected with DENV (top, patient identification numbers); numbers in centers indicate the number of antibodies from each patient. One copy of three duplicate antibodies (one EDE1 mAb and two EDE2 mAbs) from patient 752 with identical amino acid sequences was excluded from this and all other analyses. 

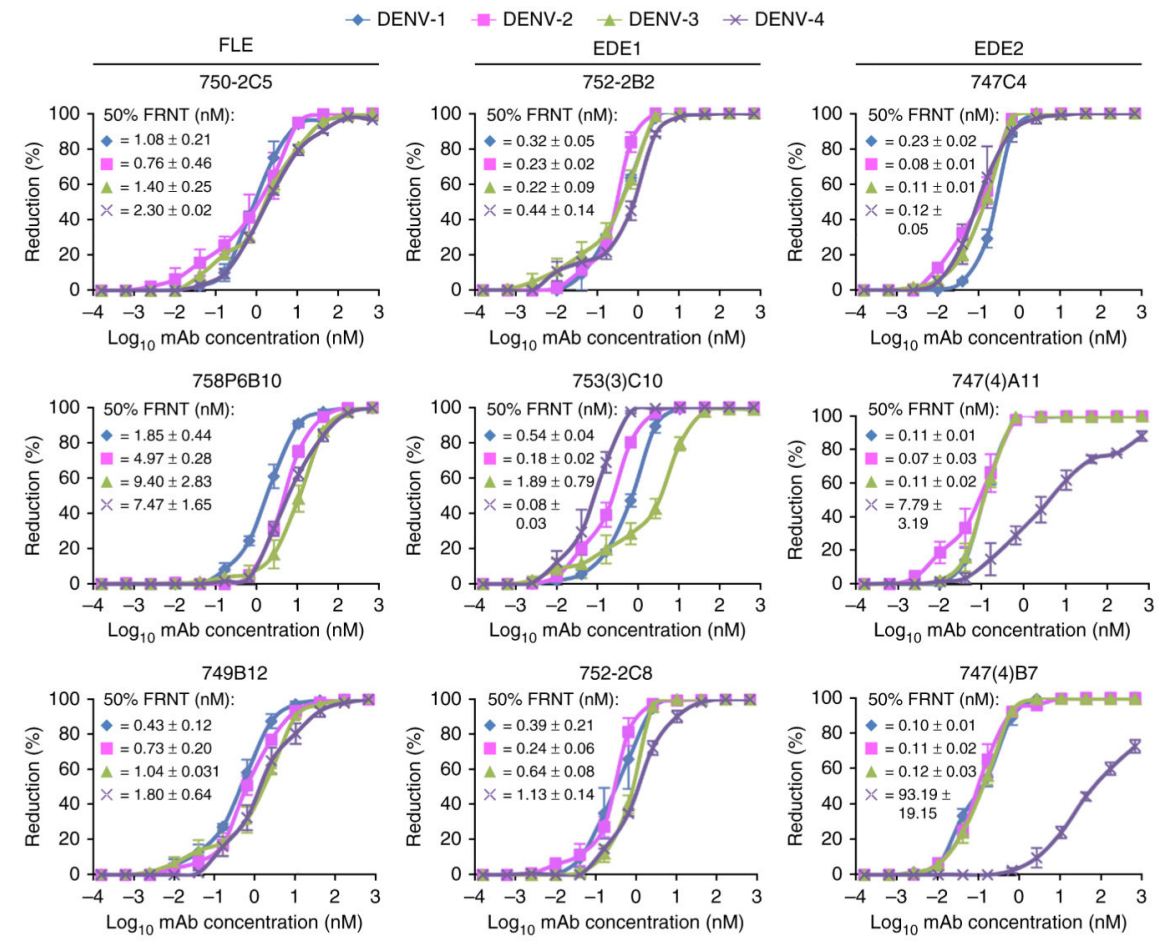

Figure 4.

Antibodies to EDE are potent and highly crossreactive in neutralization assays.

Neutralization assays on Vero cells for nine representative mAbs (identifiers above plots) to FLE, EDE1 and EDE2 (top) of all four DENV serotypes (key) produced in C6/36 insect cells, presented as the $50 \%$ focus reduction neutralization titer (FRNT). Data are from two or three independent experiments, each with duplicate wells (mean \pm s.e.m.). 
a

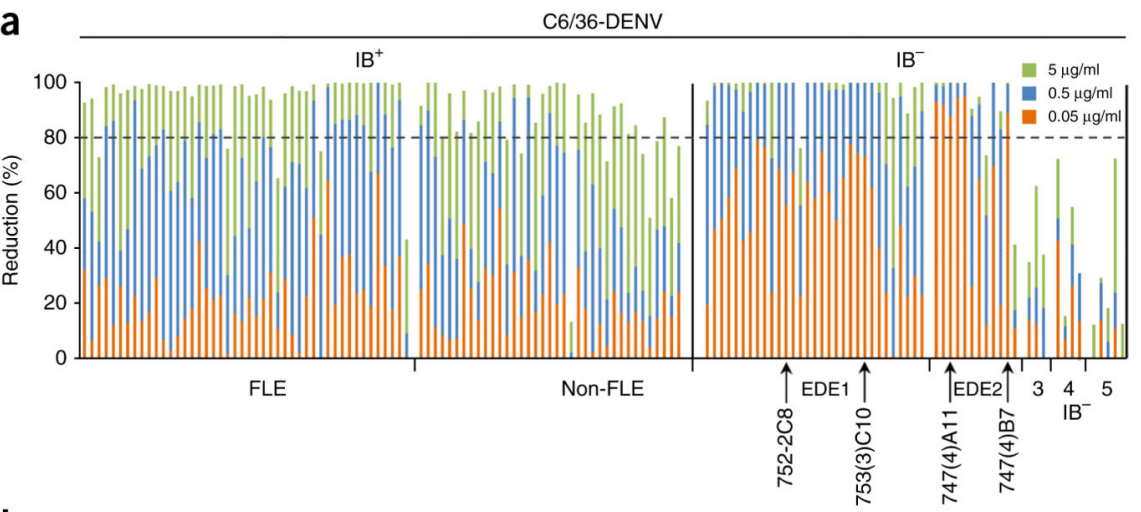

b

DC-DENV

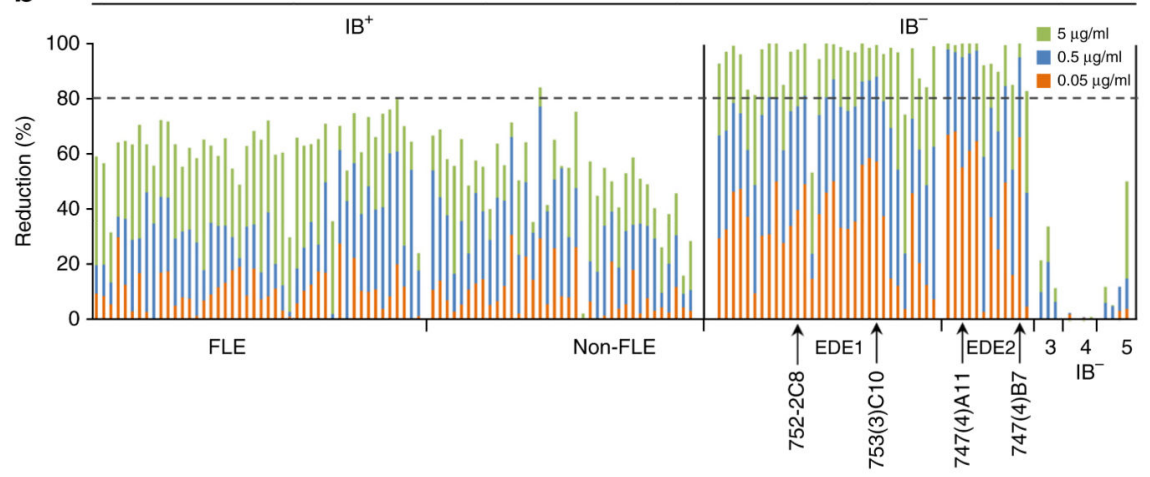

Figure 5.

EDE-specific antibodies have superior neutralizing activities. Neutralization of C6/36DENV (a) or DC-DENV (b) by $138 \mathrm{mAbs}$ (7 mAbs excluded because they did not react to DENV-2) at a final concentration of $0.05,0.5$ or $5 \mu \mathrm{g} / \mathrm{ml}$ (key (FRNT)), with Vero cells as the target. Below, classification of antibodies as FLE, EDE (five subgroups based on the results of VLP mapping: EDE1, EDE2, IB-3, IB-4 and IB-5) or non-FLE (IB ${ }^{+}$antibodies that failed to map on the VLP); arrows indicate mAbs to EDE used elsewhere ${ }^{33}$. Data represent two independent experiments each with duplicate wells. 

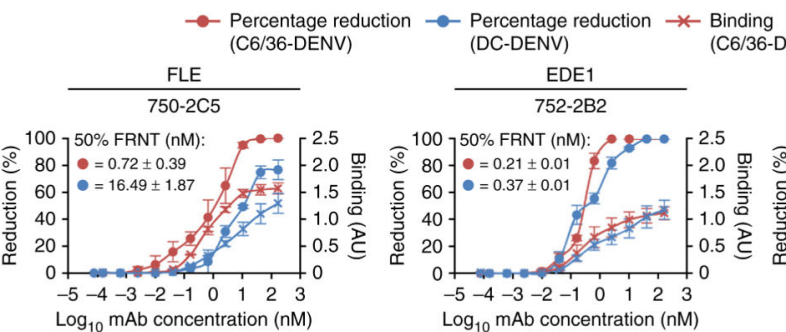

$\log _{10}$ mAb concentration (nM)

$$
758 \mathrm{p} 6 \mathrm{~B} 10
$$
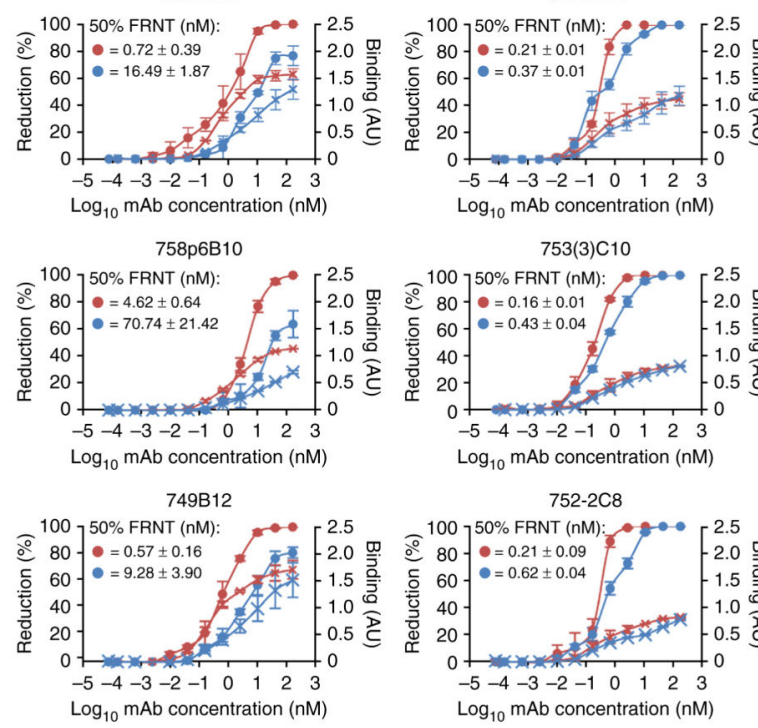

$\log _{10} \mathrm{mAb}$ concentration (nM)

753(3)C10

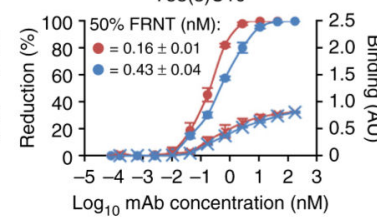

752-2C8

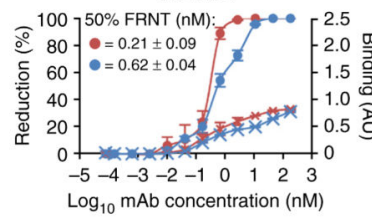

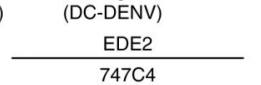

$747 C 4$

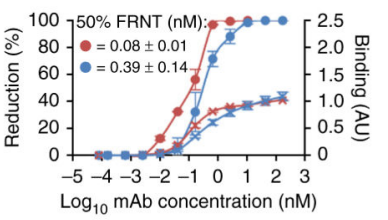

747(4)A11

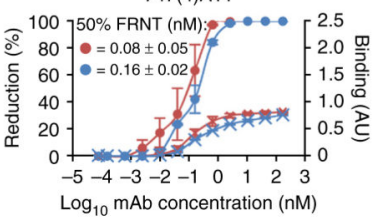

747(4)B7

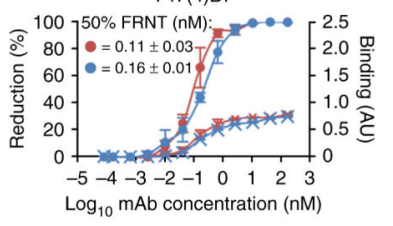

Figure 6.

Binding and neutralization of virus generated by insect cells and DCs. Titration curves for binding, measured by capture ELISA, and neutralization of DC-DENV and C6/36-DENV by three mAbs each from the FLE and EDE1 and EDE2 groups (presented as 50\% FRNT values). AU, arbitrary units. Data are from two independent experiments representative of nine experiments each with anti-FLE and anti-EDE1 and seven experiments with anti-EDE2 (mean \pm s.e.m.). 


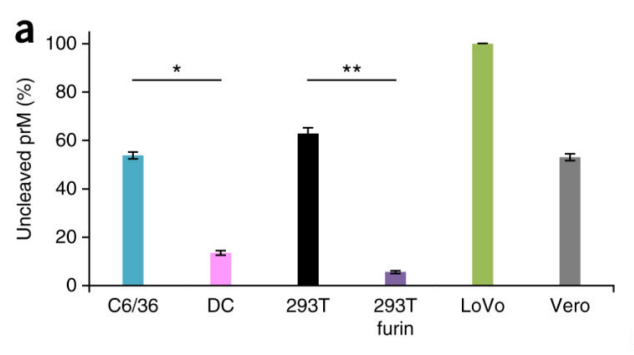

b
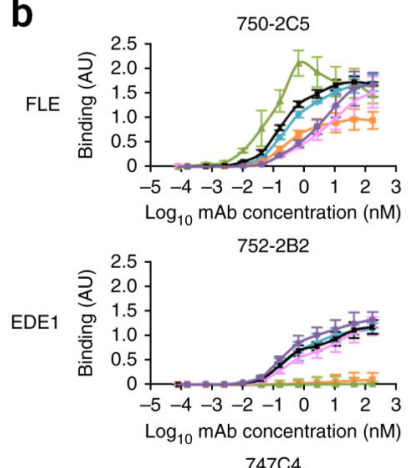

$$
\text { EDE2 }
$$
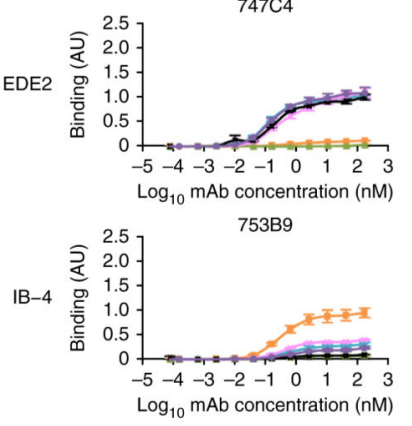
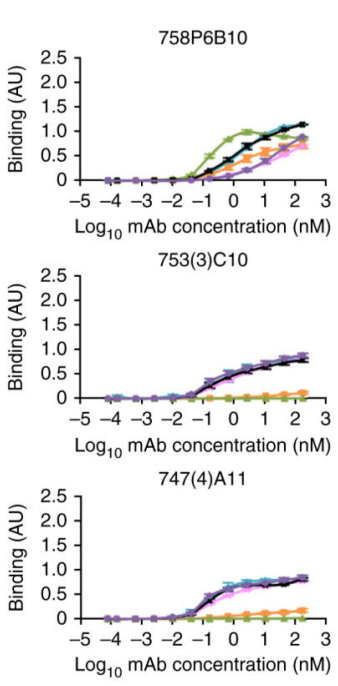

$$
\begin{array}{ll}
\rightarrow-\text { C6/36-DENV } & \rightarrow \text { 293T-DENV } \\
-\rightarrow \text { Acid-C6/36-DENV } & \rightarrow-\text { Furin-293T-DENV } \\
\rightarrow \text { DC-DENV } & \rightarrow-\text { LoVo-DENV }
\end{array}
$$

Figure 7.

Binding of antibody to viral particles in various states of maturation. (a) ELISA of anti-prM and anti-E protein, to calculate a ratio of prM to E protein on viral particles from various cells (horizontal axis); results are presented relative to those of virus from LoVo cells, set as $100 \%$ (prM content). Each new batch of virus was tested, and the data were pooled (batches of viral preparations tested: 12 (C6/36), 21 (DC), 6 (293T), 5 (293T furin), 8 (LoVo) and 8 (Vero)). $* P=0.0084$ and $* * P=0.0005$ (one-way analysis of variance (ANOVA), KruskalWallis test). (b) Capture ELISA of the binding of mAbs to DENV-2 produced from C6/36, DC, 293T cells, furin-transfected 293T cells, LoVo cells or acid-treated DENV-2. Data are representative of two experiments with three mAbs to FLE, three EDE1 mAbs and three EDE2 mAbs, representative of eight mAbs to FLE, ten EDE1 mAbs and eight EDE2 mAbs (mean \pm s.e.m.). 
a

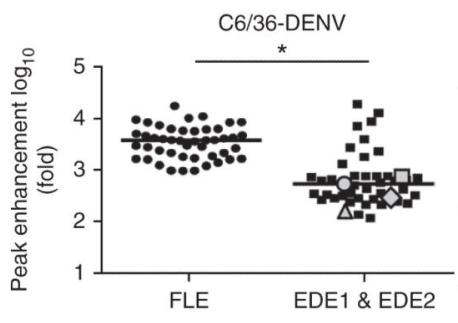

b
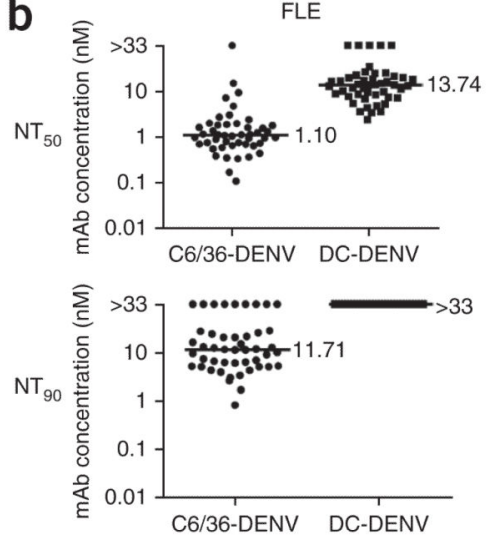
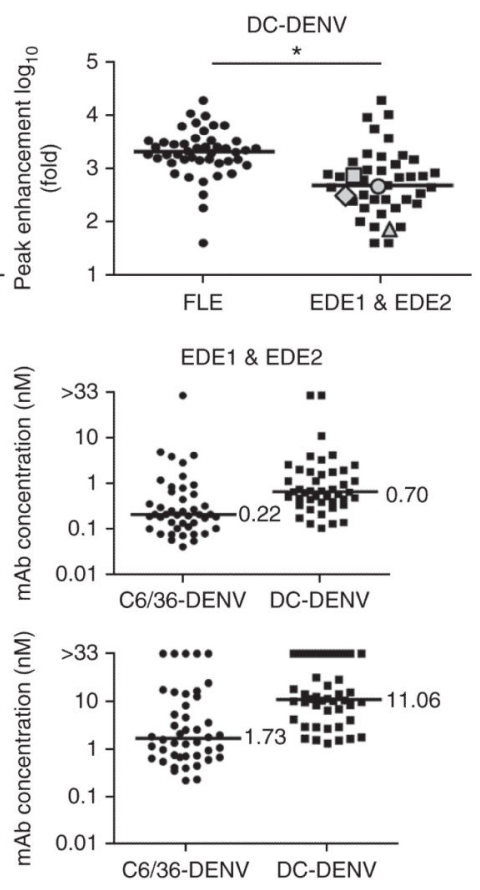

Figure 8.

Reduced ADE with mAbs to EDE. (a) ADE assays on U937 cells infected with DENV-2 grown in either $\mathrm{C} 6 / 36$ cells or DCs, in the presence of titrations of mAbs to E protein that react to FLE or EDE; results are presented as median peak enhancement (fold). $* P<0.0001$ (Mann-Whitney test). (b) $\mathrm{NT}_{50}$ and $\mathrm{NT}_{90}$ values for $\mathrm{mAbs}$ to FLE and EDE of C6/36DENV and DC-DENV. Each symbol represents an individual mAb (black symbols, this study; gray symbols, from ref. 33: diamonds, mAb 752-2C8; triangles, mAb 753(3)C10; circles, mAb 747(4)A11; squares, 747(4)B7); small horizontal lines indicate the mean. Data are representative of two experiments. 